\title{
Construction and Applicaton of Grey Concept Lattices
}

\author{
Qiang WU \\ Department of Computer Technology and Science, Shaoxing University \\ Shaoxing, 312000, China \\ e-mail: cswq@usx.edu.cn
}

Received: August 2010; accepted: October 2012

\begin{abstract}
Grey numbers facilitate the representation of uncertainty not only for elements of a set, but also the set itself as a whole. This paper utilizes the notion of possibility degree from grey system theory coupled with the idea of dominance relation and partial order set (poset) from rough theory to represent uncertain information in a manner that maintains the degree of uncertainty of information for each tuple of the original data. Concept lattices of grey information system are constructed and a decision-making algorithm that combines with grey relational grade is described. A case study is used to demonstrate the supplier selection problem applying the proposed method. The research has concluded that the method is appropriate to use.
\end{abstract}

Keywords: grey information system, grey dominance relation, poset, concept lattice, decisionmaking.

\section{Introduction and Problem Statement}

Formal concept analysis, also known as concept lattice, was proposed by Wille (1982) in early eighties. As an effective tool for knowledge discovery, concept lattice has been widespread concern in artificial intelligence researchers. In the concept lattice structure, rule extraction, application of concept lattices and so on, it has been made a series of research results (Valtchev et al., 2002; Tonella, 2003; Liang and Wang, 2004; Qu and Zhai, 2006). The traditional concept lattice is based on the binary formal context to describe the links between objects and attributes, describe the relationship of generalization and specialization between the concepts, and then all attributes are treated equally. However, in practical problems, many information systems are based on the grey relations. This information system generally implies an order of features such as large and small, high and low, so more with less. Knowledge acquisition research of information systems based on grey relations is of great significance.

Deng (1982) developed the grey system theory and presented grey decision-making systems (Deng, 1989). Grey system theory is a mathematical tool of handling missing data, uncertainty information. In recent years, many authors investigated grey system theory and its application in decision-making. To explore a more effective method to study the information content of grey numbers, an axiomatic approach was used (Liu and 
Lin, 2006). They introduced a new definition for information content of grey numbers and proved relevant results. This work fills the vacancy on how to measure the information value contained in a grey number. Multi-attribute models for grey relationships were constructed (Olson and $\mathrm{Wu}, 2006)$. It demonstrated how simulation can be used to reflect fuzzy inputs, which allows more complete probabilistic interpretation of model results. A hybrid normalized multi-attribute decision-making model for evaluating and selecting the vendor using Analytical Hierarchy Process and Fuzzy Analytical Hierarchy Process and an integrated approach of GRA (Grey Relational Analysis) to a Supply Chain model was developed (Noorul Haq and Kannan, 2007). Lin and Lee (2008) proposed a novel forecasting model. Their work modifies the algorithm of the grey forecasting model to enhance the tendency catching ability. Huang et al. (2008) examined the potentials of the software effort estimation model by integrating a genetic algorithm (GA) to the GRA. Experimental results showed that the proposed method presents more precise estimates over the results using other methods. Lin et al. (2008) presented an illustrative example of subcontractor selection by applying grey TOPSIS method. Lin et al. (2007) proposed a grey-based rough set approach to deal with the supplier selection in supply chain management. The proposed approach takes advantage of mathematical analysis power of grey system theory while at the same time utilizing data mining and knowledge discovery power of rough set theory. Wang et al. (2009a) expanded the decision model of grey target into some situation under which the decision information and target weights are the interval numbers(grey number) at the same time. However, there is usually some uncertainty involved in all multi-attribute model inputs. Zavadskas et al.'s (2009) research is to demonstrate how simulation can be used to reflect fuzzy inputs, which allows more complete interpretation of model results. In the grey rough sets, Wu and Liu (2009) studied concept lattices with interval gray number, but its lattice structure and calculation method are very complicated.

As the multi-criteria decision analysis, decision table attribute value set is often a totally ordered set. For such a decision table, the traditional theory of formal concept analysis is difficult to find the concept of the structure under the inclusion relation, which is very detrimental to knowledge discovery.

In this paper, grey formal context based on grey dominance relations was proposed. We defined the attribute posets, the object posets, then discussed some of their properties. On this basis, grey concept lattice was defined. Its construction method was given. These results are beneficial to the formal concept analysis and grey information system for further research and application.

\section{Grey Information System and Grey Dominance Relation}

As the objective and subjective conditions and random disturbances in the data acquisition, people often get the data "approximation". Many times the object has similar values. These values are often difficult to determine choice. So sometimes the part of some attribute value can not be determined, but you can know the range. If similar data can be the 


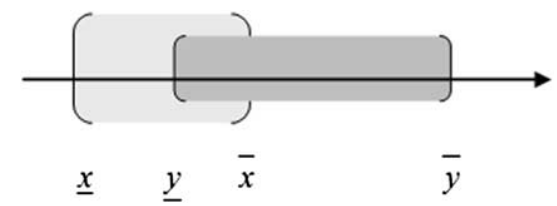

Fig. 1. Relationship of grey number (Mi et al., 2006).

same as the object of "property value" the object may take on a grey value attribute set. In addition, due to its own characteristics, some objects' attribute values have to take a grey number. For example, consider the default value, in order to facilitate the discussion, but also without loss of generality, it can usually be said for the entire range of a set. The grey numbers facilitate the representation of uncertainty not only for elements of a set, but also the set itself as a whole.

A grey number (Lin et al., 2004) is a number whose exact value is unknown, but a range within which the value lies in is known. There are the several types of grey numbers:

- Grey numbers with only lower limits: $\otimes x \in[\underline{x}, \infty)$ or $\otimes(\underline{x})$, where a fixed real value $\underline{x}$ represents the lower limit of the grey number $\otimes x$.

- Grey numbers with only upper limits: $\otimes x \in(-\infty, \bar{x}]$ or $\otimes(\bar{x})$, where $\bar{x}$ is a fixed real number or an upper limit of the grey number $\otimes x$.

- Interval grey number is the number with both lower limit $\underline{x}$ and upper limit $\bar{x}$ : $\otimes x \in[\underline{x}, \bar{x}]$.

- Continuous grey numbers and discrete grey numbers. The grey numbers continuously taking values, which cover an interval, are continuous. The grey numbers taking a finite number of values or a countable number of values in an interval are called discrete.

- Black and white numbers. When $\otimes x \in(-\infty,+\infty)$, i.e., when $\otimes x$ has neither upper nor lower limits, or the upper and the lower limits are all grey numbers, $\otimes x$ is called a black number. When $\otimes x \in[\underline{x}, \bar{x}]$ and $\underline{x}=\bar{x}, \otimes x$ is called a white number.

In the future, if not do a special note, we call grey number of the form $\otimes x \in[\underline{x}, \bar{x}]$, that interval grey number. Relationship of grey number is presented in Fig. 1.

Definition 1. A length of grey number $\otimes x$ is defined as $L(\otimes x)=\bar{x}-\underline{x}$.

Definition 2. For two grey numbers $\otimes x \in[\underline{x}, \bar{x}]$ and $\otimes y \in[y, \bar{y}]$, the possibility degree of that $\otimes x$ is less (greater) than or equal $\otimes y$ can be expressed as follows (Shi et al., 2005):

$$
\rho=\frac{\operatorname{Max}(0, L-\operatorname{Max}(0, \bar{x}-\underline{y}))}{L},
$$

where $L=L(\otimes x)+L(\otimes y)$. 
The relationship between $\otimes x$ and $\otimes y$ is determined as follows:

(1) If $\underline{x}=\underline{y}$ and $\bar{x}=\bar{y}$, we say that $\otimes x$ is equal to $\otimes y$, denoted as $\otimes x=\otimes y$, when $\rho=0.5$.

(2) If there is the intersection, when $\rho>0.5$, we say that $\otimes y$ is greater than $\otimes x$, denoted as $\otimes x \prec \otimes y$. When $\rho<0.5$, we say that $\otimes y$ is less than $\otimes x$, denoted as $\otimes x \succ \otimes y$.

Grey number is the basic unit of grey system and the operations of grey numbers are different from regular interval numbers. Interval number refers to a special one in grey number conception terms (Xie and liu, 2011). It's easy to prove the following results.

Theorem 1. Let $\otimes x \in[\underline{x}, \bar{x}], \otimes y \in[\underline{y}, \bar{y}]$ and $\otimes z \in[\underline{z}, \bar{z}]$ be grey numbers. If $L(\otimes x)$, $L(\otimes y)$ is not 0 at the same time, then the possibility degree has the following properties:

(1) $0 \leqslant \rho(\otimes x \preceq \otimes y) \leqslant 1$;

(2) $\rho(\otimes x \preceq \otimes y)=1$ iif $\bar{x} \leqslant y$;

(3) $\rho(\otimes x \preceq \otimes y)=0$ iif $\underline{x} \geqslant \overline{\bar{y}}$;

(4) (Complementation) $\rho(\otimes x \preceq \otimes y)+\rho(\otimes y \preceq \otimes x)=1$, especially $\rho(\otimes x \preceq \otimes x)=$ $\frac{1}{2}$

(5) (Transitive) $\rho(\otimes z \preceq \otimes x) \geqslant 0.5$ if $\rho(\otimes y \preceq \otimes x) \geqslant 0.5, \rho(\otimes z \preceq \otimes y) \geqslant 0.5$.

Proof. Easy to show that several other conclusions. Here, there are only (2) and (5).

(2) Since $\rho(\otimes x \preceq \otimes y)=1$, there must exist $\frac{\operatorname{Max}(0, L-\operatorname{Max}(0, \bar{x}-\underline{y}))}{L}=1$. So, $L-$ $\operatorname{Max}(0, \bar{x}-\underline{y})=L \Rightarrow \bar{x}-\underline{y} \leqslant 0 \Rightarrow \bar{x} \leqslant \underline{y}$. If $\bar{x} \leqslant \underline{y}$, then $\bar{x}-\underline{y} \leqslant 0$, we get $L-\operatorname{Max}(0, \bar{x}-\underline{y})=L \geqslant 0, \bar{\rho}=1$.

(5) In fact, $\rho(\otimes y \preceq \otimes x) \geqslant 0.5$, that is, $\frac{\operatorname{Max}\left(0, L_{1}-\operatorname{Max}(0, \bar{x}-\underline{y})\right)}{L_{1}} \geqslant 0.5, L_{1}=\bar{x}-\underline{x}+$ $\bar{y}-\underline{y}$. It must meet $\bar{x}-y \leqslant 0$ or $0 \leqslant \bar{x}-\underline{y} \leqslant \frac{1}{2} L_{1}$. Similarly, since $\rho(\otimes z \preceq \otimes y) \geqslant 0.5$, we have $\bar{y}-\underline{z} \leqslant 0$ or $0 \leqslant \bar{y}-\underline{z} \leqslant \frac{1}{2} L_{2}, \bar{L}_{2}=\bar{y}-\underline{y}+\bar{z}-\underline{z}$.

When $\bar{x}-\underline{y} \leqslant 0$ and $\bar{y}-\underline{z} \leqslant 0$, we can get $\bar{x} \leqslant \underline{z} . \rho(\otimes z \preceq \otimes x)=1 \geqslant 0.5$ by (2).

When $\bar{x}-\underline{y} \leqslant 0$ and $\bar{y}-\underline{z} \leqslant \frac{1}{2} L_{2}$, we have $\bar{y}-\underline{z} \leqslant \bar{z}-\underline{y}$. This shows that $[\underline{z}, \bar{z}]$ at $[y, \bar{y}]$ to the right. So, $\bar{x}-\underline{z} \leqslant 0$, that is, $\rho(\otimes z \preceq \otimes x)=1 \geqslant 0.5$ by (2).

When $0 \geqslant \bar{x}-y \leqslant \frac{1}{2} L_{1}$ and $\bar{y}-\underline{z} \leqslant 0$, it is easy to obtain $\bar{x}-y \leqslant \bar{y}-\underline{x}$. This shows that $[\underline{x}, \bar{x}]$ at $[\underline{y}, \bar{y}]$ to the left. And $\bar{y}-\underline{z} \leqslant 0$, so $\rho(\otimes z \preceq \otimes x)=1 \geqslant 0.5$ by (2).

When $0 \geqslant \bar{x}-\underline{y} \leqslant \frac{1}{2} L_{1}$ and $\bar{y}-\underline{z} \leqslant \frac{1}{2} L_{2}$, there exist $\bar{x}-\underline{y} \leqslant \bar{y}-\underline{x}$ and $\bar{y}-\underline{z} \leqslant \bar{z}-\underline{y}$. This shows that $[\underline{x}, \bar{x}]$ at $[\underline{y}, \bar{y}]$ to the left. And $[\underline{y}, \bar{y}]$ at $[\underline{z}, \bar{z}]$ to the left, so $\rho(\otimes z \preceq \otimes x)=$ $1 \geqslant 0.5$ by (2).

Theorem 2. A grey number set based on possibility degree is a totally ordered set.

Proof. A set is a totally ordered set in which every nonempty subset has a smallest element with the property that there is no element in the subset less than this smallest element. Firstly, from (1) and (2) in Theorem 1, every element can be compared with others in grey number set. Assume there are three grey numbers: $\otimes x, \otimes y$ and $\otimes z, \otimes x \preceq \otimes y$ and $\otimes y \preceq \otimes z$. From (5) in Theorem 1, we get $\otimes x \preceq \otimes z$. 
DEFINITION 3. Let $G S=\left(U, A, V, f_{\otimes}\right)$ denote an information system called a grey information system (Li et al., 2008), where

- $U$ : a set of objects called the universe,

- $A$ : a set of attributes,

- $V=\cup_{a \in A} V_{a}: V_{a}$ is a describing universe of the attribute $a$,

- $f_{\otimes}: U \times A \rightarrow V$, the objects describing function, which gives each object in $U$, corresponding to an attribute in $A$, a grey description.

The uncertainty of the information system can be expressed as grey number set by the describing function, that is, $\forall a \in A, u \in U, f_{\otimes}(u, a) \in V_{a}$, where $f_{\otimes}(u, a)$ is a grey number for an object $u$ at the attribute $a$, denoted by $f_{\otimes}(u, a) \in$ $\left[f_{\otimes}(u, a), \overline{f_{\otimes}}(u, a)\right]$.

In a grey information system $G S=\left(U, A, V, f_{\otimes}\right), a \in A, u \in U$, if the attribute value is completely unknown, it can be an interval grey set $\left(-\infty, V_{a}\right]$ that means that the attribute value is " $*$ " in the traditional incomplete information systems. Therefore, $\underline{f_{\otimes}}(u, a) \neq \emptyset$, and $\left(-\infty, V_{a}\right]$ has the maximum grey that indicates it could range from any subset of the domain $V_{a}$.

Definition 4. Let $S=\left(U, A, V, f_{\otimes}\right)$ be a grey information system, $U=\left\{x_{1}, x_{2}, \ldots, x_{n}\right\}$, $A=\left\{a_{1}, a_{2}, \ldots, a_{m}\right\}$. The values of object $x_{i}, x_{j} \in U$ at the corresponding attribute $a_{k} \in A$ are $f_{\otimes}\left(x_{i}, a_{k}\right)$ and $f_{\otimes}\left(x_{j}, a_{k}\right)$. If $\rho\left\{f_{\otimes}\left(x_{i}, a_{k}\right) \preceq f_{\otimes}\left(x_{j}, a_{k}\right)\right\} \geqslant(\leqslant) 0.5$, then it is denoted as $f_{\otimes}\left(x_{i}, a_{k}\right) \preceq(\succeq) f_{\otimes}\left(x_{j}, a_{k}\right)$.

DEFINITION 5. For $G S=\left(U, A, V, f_{\otimes}\right)$, the values of objects $x, y \in U$ at corresponding attribute $a \in B \subseteq A$ are $f_{\otimes}(x, a)$ and $f_{\otimes}(y, a), B \subseteq A$ identifies a binary relation $R$ in the $U$ as follows:

$$
\begin{aligned}
& R_{\bar{B}}^{\prec}=\left\{(x, y) \in U^{2} \mid f_{\otimes}\left(x_{i}, a_{k}\right) \preceq f_{\otimes}\left(x_{j}, a_{k}\right),(a \in B)\right\}, \\
& R_{\bar{B}}^{\succ}=\left\{(x, y) \in U^{2} \mid f_{\otimes}\left(x_{i}, a_{k}\right) \succeq f_{\otimes}\left(x_{j}, a_{k}\right),(a \in B)\right\} .
\end{aligned}
$$

$R$ is called the grey dominance relation in grey information system $G S=\left(U, A, V, f_{\otimes}\right)$. $(x, y) \in R_{\bar{B}}^{\prec}$, the object $y$ contains the object $x$ in $B ;(x, y) \in R_{\bar{B}}^{\succ}$ said the object $y$ on the attribute set $B$ is contained in the object $x$.

\section{Poset and FCA}

The term "poset" is short for "partially ordered set", that is, a set whose elements are ordered but not all pairs of elements are required to be comparable in the order.

DEFINITION 6. A poset is a pair $(P, \preceq)$, where $P$ is a set, and $\preceq$ is a binary relation on $P$ (i.e., $\preceq$ is a subset of $P \times P$ ) which is 
(1) Reflexive ( $x \preceq x$ for all $x \in P$ ),

(2) Anti-symmetric ( $x \preceq y$ and $y \preceq x \Rightarrow x=y$ for all $x, y \in P$ ),

(3) Transitive ( $x \preceq y$ and $y \preceq z \Rightarrow x=z$ for all $x, y, z \in P$; Trotter, 1995).

In a grey information system $G S=\left(U, A, V, f_{\otimes}\right)$, a grey dominance relation $R$ on $U$ determined by $B \subseteq A$ satisfies the following properties:

(1) $R$ is reflexive and anti-symmetric, and transitive, so it is the partial order;

(2) $R_{\bar{B}_{1}}^{\prec} \subseteq R_{\bar{B}_{2}}^{\prec} \subseteq R_{\bar{A}}^{\swarrow}, R_{\bar{B}_{1}}^{\succ} \subseteq R_{\bar{B}_{2}}^{\succ} \subseteq R_{\bar{A}}^{\succ}$, if $B_{1} \subseteq B_{2} \subseteq A$. Let $[x]_{\bar{B}}^{\prec}=\{y \mid(x, y) \in$ $\left.R_{\bar{B}}^{\succ}\right\},[x]_{\bar{B}}^{\succ}=\left\{y \mid(x, y) \in R_{\bar{B}}^{\succ}\right\},[x]_{B}^{\prec}$ and $[x]_{B}^{\succ}$ are called a dominance class and an inferior class of $x$ in the $B$, respectively.

The following are quoted from reference (Ganter and Wille, 1999).

Definition 7. Let $(P, \preceq)$ be a poset, $B \subseteq P . s \in P$ is a infimum (a unique greatest lower bound), if $s \preceq a$ for $a \in B$. We simply write $\wedge B$. The definition of supremum (a unique least upper bound) is similar. Its written as $\vee B$.

DEFINITION 8. A lattice is a poset denoted by $(P, \preceq)$ in which each pair of elements has a unique supremum and a unique infimum. A lattice is complete if there exists a supremum and infimum for every one of its subsets.

Definition 9. A formal context is a triple $(U, A, I)$ consisting of two sets $U=$ $\left\{x_{1}, x_{2}, \ldots, x_{n}\right\}$ and $A=\left\{a_{1}, a_{2}, \ldots, a_{n}\right\}$ and a relation $I$ between $U$ and $A(I \in$ $U \times A)$. The elements of $U$ are called objects and the elements of $A$ are called attributes. $I$ is called the incidence relation and describes whether an object in $U$ has a specific attribute in $A$. For $X \subseteq V$ and $Y \subseteq A$, define $X^{*}=\{a \in A \mid \forall x \in$ $X,(x, a) \in I\}, Y^{*}=\{x \in U \mid \forall a \in Y,(x, a) \in I\}$. In particular, for $x \in U$ and $a \in A, x^{*}=\{x\}^{*}, a^{*}=\{a\}^{*}$.

Definition 10. For $X \subseteq V$ and $Y \subseteq A$, a concept of context $K=(U, A, I)$ is a pair $(X, Y)$ where $X^{*}=Y$ and $Y^{*}=X . X$ is called the extent of the concept and $Y$ is called the intent. With $L(K)$ to represent the set of all concepts of $K$.

DEFINITION 11. For concepts $C_{1}=\left(X_{1}, Y_{1}\right)$ and $C_{2}=\left(X_{2}, Y_{2}\right)$, we write $\left(X_{1}, Y_{1}\right) \preceq$ $\left(X_{2}, Y_{2}\right)$ iff $X_{1} \subseteq X_{2}$ (and dually $\left.Y_{1} \subseteq Y_{2}\right)$. The set of concepts ordered by the relation $\preceq$ forms a complete lattice called a concept lattice.

Obviously, relations “ $\preceq$ ” is a partial order on $L(K)$. It can be induced a lattice structure. It can be shown that it is a complete lattice. These meet and join operations of concept lattices are defined as follows:

$$
\begin{aligned}
& \left(X_{1}, Y_{1}\right) \wedge\left(X_{2}, Y_{2}\right)=\left(X_{1} \cap X_{2},\left(Y_{1} \cup Y_{2}\right)^{* *}\right), \\
& \left(X_{1}, Y_{1}\right) \vee\left(X_{2}, Y_{2}\right)=\left(\left(X_{1} \cup X_{2}\right)^{* *}, Y_{1} \cap Y_{2}\right) .
\end{aligned}
$$

Formal concept analysis (FCA) on the detailed description, see reference (Ganter and Wille, 1999). 


\section{Concept Lattice Based on Grey Dominance Relation}

Definition 12. A grey formal context is a triple $G S K=\left(U, V_{A}, I\right)$, where $V_{A}=$ $\left\{V_{a}\right\}, a \in A$. $V_{a}$ is a set of $a_{i}$ attribute values. $I$ is a binary relation between $U$ and $V_{a}$, if $f_{\otimes}(x, a) \succeq(\preceq) f_{\otimes}(y, a)$ for $\forall f_{\otimes}(x, a), f_{\otimes}(y, a) \in V_{a}$.

In grey formal context, the attribute set is divided into several blocks, each block on behalf of grey describing domain of an attribute. Therefore, in the same grey describing domain of the two attributes, $f_{\otimes}(x, a) \in V_{a}$ and $f_{\otimes}(y, a) \in V_{a}$, is comparable, otherwise there is no dominance relation between attributes. Attribute poset is denoted by $\left(V_{A}, \preceq\right)$.

DEFInition 13. Let $G S K=\left(U, V_{A}, I\right)$ be a grey formal context. $V_{A}=\cup_{a \in A} V_{a}$, $x_{1}, x_{2} \in U, f_{\otimes}\left(x_{1}, a\right), f_{\otimes}\left(x_{2}, a\right) \in V_{A}$. The partial order relation on objects set $U$ is defined as

$$
x_{1} \preceq x_{2} \quad \Leftrightarrow \quad\left\{\left(x_{1}^{*} \cap V_{a}\right) \preceq\left(x_{1}^{*} \cap V_{a}\right) \mid \forall V_{a} \in V_{A}\right\} .
$$

In our study, $(U, \preceq)$ is used to represent objects poset.

DEFINITION 14. Let $G S K=\left(U, V_{A}, I\right)$ be a grey formal context. For any $x, y \in$ $U, f_{\otimes}(x, a), f_{\otimes}(y, a) \in V_{A}, B \subseteq A$

(1) If there exists $\left(x^{*} \cap V_{a}\right) \preceq\left(y^{*} \cap V_{a}\right)$ such that $[x] \frac{\preceq}{B}=\left\{y \in U \mid\left(x^{*} \cap V_{a}\right) \preceq\right.$ $\left.\left(y^{*} \cap V_{a}\right), \forall V_{A} \in V_{B}\right\}$, then $[x]_{B}^{\prec}$ is a dominance class of object $x$.

(2) If there exists $f_{\otimes}(y, a) \preceq f_{\otimes}(x, a), a \in B \subseteq A, x, y \in U$ such that $\left[f_{\otimes}(x, a)\right]_{B}=\left\{f_{\otimes}(y, a) \mid f_{\otimes}(y, a) \preceq f_{\otimes}(x, a), a \in B, x, y \in U\right\}$, then $\left[f_{\otimes}(x, a)\right]_{B}$ is a dominance class of attribute $a$.

Theorem 3. Let $G S K=\left(U, V_{A}, I\right)$ be a grey formal context. If $C=\left\{f_{\otimes}(x, a) \mid f_{\otimes}(x, a) \preceq\right.$ $\left.f_{\otimes}\left(x_{1}, a\right), f_{\otimes}(x, a) \in V_{A}\right\}, B=\left\{f_{\otimes}(x, a) \mid f_{\otimes}(x, a) \preceq f_{\otimes}\left(x_{2}, a\right), f_{\otimes}(x, a) \in V_{A}\right\}$, for $x_{1}, x_{2} \in U$, then

(1) $x_{1} \preceq x_{2} \Leftrightarrow C \subseteq B$.

(2) $x_{2} \in\left[x_{1}\right] \frac{\preceq}{B} \Leftrightarrow C \subseteq B$.

Proof.

(1) Since $x_{1} \preceq x_{2} \Leftrightarrow\left\{\left(x_{1}^{*} \cap V_{a}\right) \preceq\left(x_{1}^{*} \cap V_{a}\right) \mid \forall V_{a} \in V_{A}\right\}$, we have $x_{1}^{*}=f_{\otimes}\left(x_{1}, a\right) \preceq$ $x_{2}^{*}=f_{\otimes}\left(x_{2}, a\right)$, for $\forall V_{a} \in V_{A}$. That is, $f_{\otimes}\left(x_{2}, a\right) \in V_{C} \Rightarrow f_{\otimes}\left(x_{1}, a\right) \in V_{B}$. Its reverse is not true. So, $C \subseteq B$.

(2) $x_{2} \in\left[x_{1}\right] \frac{\preceq}{B}$, that is, $x_{1} \preceq x_{2}$. The proof is same as (1). 
Definition 15. Let $G S K=\left(U, V_{A}, I\right)$ be the grey formal context, $X \subseteq U, Y \subseteq A$. Then grey operating of $X$ and $Y$ are defined by its two mappings $X^{\prime}$ and $Y^{\prime}$.

$$
\begin{gathered}
X^{\prime}=\bigcap\left\{x ^ { * } \cup \left\{f_{\otimes}(y, a) \mid f_{\otimes}(y, a) \preceq f_{\otimes}(x, a), f_{\otimes}(y, a) \in V_{a},\right.\right. \\
\left.\left.f_{\otimes}(x, a) \in x^{*}\right\}, x \in X\right\}, \\
Y^{\prime}=\bigcap\left\{f _ { \otimes } ( x , a ) ^ { * } \cup \left\{\left(f_{\otimes}(y, a)\right)^{*} \mid f_{\otimes}(y, a) \succeq f_{\otimes}(x, a),\right.\right. \\
\left.\left.f_{\otimes}(y, a) \in V_{a}\right\}, f_{\otimes}(x, a) \in Y\right\} . \\
x^{\prime}=\{x\}^{\prime}, f_{\otimes}(x, a)^{\prime}=\left\{f_{\otimes}(x, a)\right\}^{\prime}, \text { for } x \in U \text { and } f_{\otimes}(x, a) \in V_{A} .
\end{gathered}
$$

DEFINITION 16. A grey (formal) concept of $G S K=\left(U, V_{A}, I\right)$ is a pair $(X, Y)$ with $X \subseteq U, Y \subseteq V_{A}, X^{\prime}=Y$ and $Y^{\prime}=X$. The sets $X$ and $Y$ are called the extent and the intent of the grey concept $(X, Y)$, respectively. The subconcept-superconcept relation is formalized by

$$
\left(X_{1}, Y_{1}\right) \preceq\left(X_{2}, Y_{2}\right) \quad \Leftrightarrow \quad X_{1} \subseteq X_{2}\left(\Leftrightarrow Y_{1} \supseteq Y_{2}\right) .
$$

The set of all grey concepts of $G S K$ together with the dominance relation is always a complete lattice, called the grey concept lattice of $G S K$ and denoted by $G L\left(U, V_{A}, I\right)$.

Theorem 4. Let $G S K=\left(U, V_{A}, I\right)$ be the grey formal context, $\forall(X, Y) \in G L\left(U, V_{A}, I\right)$, then the total number of all attributes including in $Y$ is $|A|$.

Proof. Since each object must have a attribute in the grey information system, $x^{*}$ involves all attributes in grey formal context. $\left|x^{*}\right|=|A| . X^{\prime}=\cap\left\{x^{*} \cup\left\{f_{\otimes}(y, a) \mid f_{\otimes}(y, a) \preceq\right.\right.$ $\left.\left.f_{\otimes}(x, a), f_{\otimes}(y, a) \in V_{a}, f_{\otimes}(x, a) \in x^{*}\right\}, x \in X\right\}$, so, $\left|x^{*}\right|$ is the total number of all attribute including in $Y$. The conclusion is true.

Grey formal concept analysis allows us to identify meaningful groupings of elements that have common dominance attributes. From the conceptual viewpoint, each grey concept represents a category of elements described by a set of dominance attributes. We will use it to decision-making.

\section{Grey Concept Lattice and Decision-Making}

Definition 17. Let $S_{g}$ be a grey relational factors set. Suppose that $x_{0} \in S_{g}$ is the reference series and $x_{1}, x_{2}, \ldots, x_{n} \in S_{g}$ are the objective series. $r\left(x_{0}, x_{i}\right)=$ $\frac{1}{m} \sum_{k=1}^{m} r\left(x_{0}(k), x_{i}(k)\right)$ is the grey relational grade between the reference series $x_{0}$ and the objective series $x_{i}(i=1,2, \ldots, n)$ at point $k \in\{1,2, \ldots, m\}$ if it satisfies:

Normality: $0<r\left(x_{0}, x_{i}\right) \leqslant 1, \forall k, r\left(x_{0}, x_{i}\right)=1 \Leftrightarrow x_{0}=x_{i}, r\left(x_{0}, x_{i}\right)=0 \Leftrightarrow$ $x_{i} \in \emptyset$. 
Entirety: $x_{i}, x_{j} \in S_{g}=\left\{S_{g}^{\sigma} \mid, \sigma=0,1, \ldots, n\right\}, n \geqslant 2, r\left(x_{j}, x_{i}\right) \neq^{\text {often }} r\left(x_{i}, x_{j}\right)$. Symmetry: $r(x, y) \equiv r(y, x)$, if $S_{g}=\{x, y\}$.

Proximity: $r\left(x_{0}(k), x_{i}(k)\right)$ increases as $\left|x_{0}(k), x_{i}(k)\right|$ decrease for $\forall k \in\{1,2, \ldots, m\}$. $r\left(x_{0}(k), x_{i}(k)\right)$ is the grey relational coefficient (Wu et al., 1996).

In the grey relational analysis, when the range of the sequence is large or the standard value is enormous, the function of factors is neglected. However, if the factors goals and directions are different, the grey relational analysis might also produce incorrect results. Therefore, one has to preprocess the data which are related to a group of series, which is called grey theory relational generation (Deng, 1990; Chang et al., 1996). It can reduce the randomization and increase the regularity of data.

Suppose that $U=\left\{X_{1}, X_{2}, \ldots, X_{l}\right\}$ is a program set that is constructed by a group grey formal concepts for decision-making. $A=\left\{a_{1}, a_{2}, \ldots, a_{m}\right\}$ is a set of attributes. A pair $\left(X_{i}, V_{A}\right)$ is a grey formal concept. Option $X_{i}$ 's attribute value at $a_{j}$ is a grey number set $f_{\otimes}\left(X_{i}, a_{j}\right)=\left\{f_{\otimes}\left(x_{i 1}, a_{j}\right), f_{\otimes}\left(x_{i 2}, a_{j}\right), \ldots, f_{\otimes}\left(x_{i s}, a_{j}\right)\right\}\left(s=\left|X_{i}\right| ; j=\right.$ $1,2, \ldots, m)$. It's attribute vector is denoted by $\left(f_{\otimes}\left(X_{i}, a_{1}\right), f_{\otimes}\left(X_{i}, a_{2}\right), \ldots, f_{\otimes}\left(X_{i}, a_{m}\right)\right)$ $(i=1,2, \ldots, n)$. In order to eliminate the dimensionless and increase the comparability, $f_{\otimes}\left(X_{i}, a_{j}\right)(i=1,2, \ldots, n ; j=1,2, \ldots, m)$ is standardized by range transform. They are described as follows:

(1) If the expectancy of the response is larger-the-better (i.e., beneficial response), then it can be expressed by

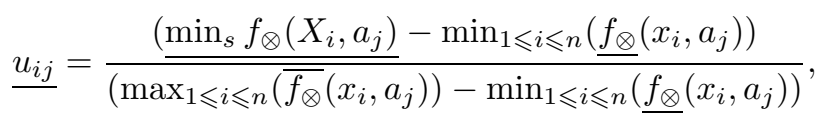

$$
\begin{aligned}
& \overline{u_{i j}}=\frac{\left(\overline{\min _{s} f_{\otimes}\left(X_{i}, a_{j}\right)}-\min _{1 \leqslant i \leqslant n}\left(\underline{f_{\otimes}}\left(x_{i}, a_{j}\right)\right)\right.}{\left(\max _{1 \leqslant i \leqslant n}\left(\overline{f_{\otimes}}\left(x_{i}, a_{j}\right)\right)-\min _{1 \leqslant i \leqslant n}\left(\underline{f_{\otimes}}\left(x_{i}, a_{j}\right)\right)\right.} .
\end{aligned}
$$

(2) If the expectancy of the response is smaller-the-better (i.e., non-beneficial response), then it can be expressed by

$$
\begin{aligned}
& \underline{u_{i j}}= \frac{\left(\max _{1 \leqslant i \leqslant n}\left(\overline{f_{\otimes}}\left(x_{i}, a_{j}\right)\right)-\overline{\left.\min _{s} f_{\otimes}\left(X_{i}, a_{j}\right)\right)}\right.}{\left(\max _{1 \leqslant i \leqslant n}\left(\overline{f_{\otimes}}\left(x_{i}, a_{j}\right)\right)-\min _{1 \leqslant i \leqslant n}\left(\underline{f_{\otimes}}\left(x_{i}, a_{j}\right)\right)\right.}, \\
& \overline{u_{i j}}=\frac{\left(\max _{1 \leqslant i \leqslant n}\left(\overline{f_{\otimes}}\left(x_{i}, a_{j}\right)\right)-\min _{s} f_{\otimes}\left(X_{i}, a_{j}\right)\right.}{\left(\max _{1 \leqslant i \leqslant n}\left(\overline{f_{\otimes}}\left(x_{i}, a_{j}\right)\right)-\min _{1 \leqslant i \leqslant n}\left(\underline{f_{\otimes}}\left(x_{i}, a_{j}\right)\right)\right.} .
\end{aligned}
$$

DEFINITION 18. Let $\otimes \mathbf{u}_{i}=\left\{\otimes u_{i 1}, \otimes u_{i 2}, \ldots, \otimes u_{i m}\right\}(i=1,2, \ldots, n)$ be evaluation vectors of the program that is standardized, where $\otimes u_{i j} \in\left[u_{i j}, \overline{u_{i j}}\right] . \otimes \mathbf{u}^{+}=$ $\left\{\otimes u_{1}^{+}, \otimes u_{2}^{+}, \ldots, \otimes u_{m}^{+}\right\}, \otimes \mathbf{u}^{-}=\left\{\otimes u_{1}^{-}, \otimes u_{2}^{-}, \ldots, \otimes u_{m}^{-}\right\}$are called the ideal optimal program effectiveness evaluation vector and the critical optimal program effectiveness evaluation vector respectively, if

$$
{\underline{u_{j}}}^{+}=\max _{1 \leqslant i \leqslant n}\left\{\underline{u_{i j}}\right\}, \quad \underline{u_{j}}{ }^{-}=\min _{1 \leqslant i \leqslant n}\left\{\underline{u_{i j}}\right\},
$$




$$
{\overline{u_{j}}}^{+}=\max _{1 \leqslant i \leqslant n}\left\{\overline{u_{i j}}\right\}, \quad{\overline{u_{j}}}^{-}=\min _{1 \leqslant i \leqslant n}\left\{\overline{u_{i j}}\right\}
$$

where $\otimes u_{j}^{+} \in\left[\underline{u}_{j}^{+}, \bar{u}_{j}^{+}\right], \otimes u_{j}^{-} \in\left[\underline{u}_{j}^{-}, \bar{u}_{j}^{-}\right], j=1,2, \ldots, m$.

DEFINITION 19. Let $\lambda \in[0,1]$ be a distinguishing coefficient, the grey relational grade $G\left(\otimes \mathbf{u}^{+}, \otimes \mathbf{u}_{i}^{+}\right)$between an objective series $\otimes \mathbf{u}_{i}$ and the reference series $\otimes \mathbf{u}^{+}$is defined as follow:

$$
G\left(\otimes \mathbf{u}^{+}, \otimes \mathbf{u}_{i}^{+}\right)=\sum_{j=1}^{m} \omega_{j} r_{i j}^{+}, \quad i=1,2, \ldots, n,
$$

where

$$
\begin{aligned}
r_{i j}^{+}=\frac{1}{2}[ & \left(\underline{\Delta}_{\min }^{+}+\lambda \underline{\Delta}_{\max }^{+}\right) /\left(\underline{\Delta}^{+}+\lambda \underline{\Delta}_{\max }^{+}\right) \\
& \left.+\left(\bar{\Delta}_{\min }^{+}+\lambda \bar{\Delta}_{\max }^{+}\right) /\left(\bar{\Delta}^{+}+\lambda \bar{\Delta}_{\max }^{+}\right)\right] .
\end{aligned}
$$

$\sum_{j=1}^{m} \omega_{j}=1, \omega_{j}$ is the weight of response $j$ and usually depends on decision makers' judgment.

In (16), $r_{i j}^{+}$is the gray relational coefficient and $\underline{\Delta}^{+}=\left|\underline{u}_{j}^{+}-\underline{u}_{i j}\right|, \bar{\Delta}^{+}=\left|\bar{u}_{j}^{+}-\bar{u}_{i j}\right|$,

$$
\begin{aligned}
& \Delta_{\min }^{+}=\min \left\{\underline{\Delta}^{+}, i=1,2, \ldots, n ; j=1,2, \ldots, m\right\}, \\
& \Delta_{\max }^{+}=\max \left\{\underline{\Delta}^{+}, i=1,2, \ldots, n ; j=1,2, \ldots, m\right\}, \\
& \bar{\Delta}_{\min }^{+}=\min \left\{\bar{\Delta}^{+}, i=1,2, \ldots, n ; j=1,2, \ldots, m\right\}, \\
& \bar{\Delta}_{\max }^{+}=\max \left\{\bar{\Delta}^{+}, i=1,2, \ldots, n ; j=1,2, \ldots, m\right\} .
\end{aligned}
$$

DEFINITION 20. Let $\lambda \in[0,1]$ be a distinguishing coefficient,

$$
G\left(\otimes \mathbf{u}^{-}, \otimes \mathbf{u}_{i}\right)=\sum_{j=1}^{m} \omega_{j} r_{i j}^{-}, \quad i=1,2, \ldots, n .
$$

$G\left(\otimes \mathbf{u}^{-}, \otimes \mathbf{u}_{i}\right)$ is the grey relational grade between $\otimes \mathbf{u}_{i}$ and $\otimes \mathbf{u}^{-}$, where

$$
\begin{aligned}
r_{i j}^{-}= & \frac{1}{2}\left[\left(\underline{\Delta}_{\min }^{-}+\lambda \underline{\Delta}_{\max }^{-}\right) /\left(\underline{\Delta}^{-}+\lambda \underline{\Delta}_{\max }^{-}\right)\right. \\
& \left.+\left(\bar{\Delta}_{\min }^{-}+\lambda \bar{\Delta}_{\max }^{-}\right) /\left(\bar{\Delta}^{-}+\lambda \bar{\Delta}_{\max }^{-}\right)\right],
\end{aligned}
$$

$\sum_{j=1}^{m} \omega_{j}=1, \omega_{j}$ is the weight.

In (18), $r_{i j}^{-}$is the gray relational coefficient and $\underline{\Delta}^{-}=\left|\underline{u}_{i j}-\underline{u}_{j}^{-}\right|, \bar{\Delta}^{-}=\left|\bar{u}_{i j}-\bar{u}_{j}^{-}\right|$,

$$
\begin{aligned}
& \underline{\Delta}_{\min }^{-}=\min \left\{\underline{\Delta}^{-}, i=1,2, \ldots, n ; j=1,2, \ldots, m\right\}, \\
& \Delta_{\max }^{-}=\max \left\{\underline{\Delta}^{-}, i=1,2, \ldots, n ; j=1,2, \ldots, m\right\},
\end{aligned}
$$




$$
\begin{aligned}
& \bar{\Delta}_{\text {min }}^{-}=\min \left\{\bar{\Delta}^{-}, i=1,2, \ldots, n ; j=1,2, \ldots, m\right\}, \\
& \bar{\Delta}_{\max }^{-}=\max \left\{\bar{\Delta}^{-}, i=1,2, \ldots, n ; j=1,2, \ldots, m\right\} .
\end{aligned}
$$

The following linear planning models are used to compute the ideal optimal and the critical optimal relational grade,respectively.

$$
\begin{aligned}
& \max G\left(\otimes \mathbf{u}^{+}, \otimes \mathbf{u}_{i}\right)=\sum_{j=1}^{m} \omega_{j} r_{i j}^{+}, \\
& \max G\left(\otimes \mathbf{u}^{-}, \otimes \mathbf{u}_{i}\right)=\sum_{j=1}^{m} \omega_{j} r_{i j}^{-} .
\end{aligned}
$$

And then, we can obtain a synthetical grey linear relational grade

$$
G\left(\otimes \mathbf{u}_{i}\right)=\beta_{1}\left[\max G\left(\otimes \mathbf{u}^{+}, \otimes \mathbf{u}_{i}\right)\right]+\beta_{2}\left[1-\max G\left(\otimes \mathbf{u}^{-}, \otimes \mathbf{u}_{i}\right)\right],
$$

where $i=1,2, \ldots, n$. $\beta_{1}, \beta_{2}$ are weights of the two relational grades, that is, $\beta_{1}+\beta_{2}=1$. Usually, there is $\beta_{1}=\beta_{2}=\frac{1}{2}$.

The algorithm of grey relational decision-making:

Step 1. Determine the grey formal context with original decision table (gray Information System) and find all formal concepts in it. Construct decision matrix of compared formal concepts.

Step 2. Normalization. The decision matrix $\Re=\left(\otimes x_{i j}\right)_{n \times m}$ is translated into the normalized one $\widetilde{\Re}=\left(\otimes u_{i j}\right)_{n \times m}$ using (10) to (13).

Step 3. Data series construction. Calculate the ideal optimal and the critical optimal series $\otimes \mathbf{u}^{+}$and $\otimes \mathbf{u}^{-}$using (14) and (15) from $\widetilde{\Re}=\left(\otimes u_{i j}\right)_{n \times m}$, respectively.

Step 4. Grey relational coefficient. Calculate the grey relational coefficient $r_{i j}^{+}, r_{i j}^{-}$ between $\otimes u_{i j}$ and the two different factors by (17) and (19).

Step 5. Grey relational grade calculation. Compute the grey relation grade of option $\otimes \mathbf{u}_{i}$ with respect to the two optimal series using the two linear planning models (20) and (21), respectively.

Step 6. Feature subset selection. Compute the grey relational grade $G\left(\otimes \mathbf{u}_{i}\right)$ of $\otimes \mathbf{u}_{i}$ using (22) for decision-making prediction purpose. Sort the grey relational grades. The features with higher score comprise the optimal feature subset.

\section{Application Examples}

We take the example (Wang et al., 2009b) as the sample to explain the validity of this method. The investment bank wants to invest in four enterprises $s_{1}, s_{2}, s_{3}, s_{4}$ in one city. Now we evaluate four indexes $a_{1}$ (net output rate of investment), $a_{2}$ (investment rate of profit and tax), $a_{3}$ (internal returns ratio), $a_{4}$ (environmental pollution degree) to decide which one to invest. 
Table 1

Original decision table

\begin{tabular}{lllll}
\hline & $a_{1}$ & $a_{2}$ & $a_{3}$ & $a_{4}$ \\
\hline$s_{1}$ & {$[1.8,2.2]$} & {$[1.2,1.8]$} & {$[1.8,2.2]$} & {$[5.4,5.6]$} \\
$s_{2}$ & {$[2.3,2.7]$} & {$[2.4,3.0]$} & {$[1.6,2.0]$} & {$[6.4,6.6]$} \\
$s_{3}$ & {$[1.6,2.0]$} & {$[1.7,2.3]$} & {$[1.9,2.3]$} & {$[4.4,4.6]$} \\
$s_{4}$ & {$[2.0,2.4]$} & {$[1.5,2.1]$} & {$[1.8,2.2]$} & {$[4.9,5.1]$} \\
\hline
\end{tabular}

Table 2

The grey formal context of Table 1

\begin{tabular}{lllllllll}
\hline & $a_{11}$ & $a_{12}$ & $a_{13}$ & $a_{14}$ & $a_{21}$ & $a_{22}$ & $a_{23}$ & $a_{24}$ \\
\hline$s_{1}$ & 0 & 1 & 0 & 0 & 1 & 0 & 0 & 0 \\
$s_{2}$ & 0 & 0 & 0 & 1 & 0 & 0 & 0 & 1 \\
$s_{3}$ & 1 & 0 & 0 & 0 & 0 & 0 & 1 & 0 \\
$s_{4}$ & 0 & 0 & 1 & 0 & 0 & 1 & 0 & 0 \\
\hline
\end{tabular}

Table 3

The grey formal context of Table 1 (continue)

\begin{tabular}{llllllll}
\hline & $a_{31}$ & $a_{32}$ & $a_{33}$ & $a_{41}$ & $a_{42}$ & $a_{43}$ & $a_{44}$ \\
\hline$s_{1}$ & 0 & 1 & 0 & 0 & 0 & 1 & 0 \\
$s_{2}$ & 1 & 0 & 0 & 0 & 0 & 0 & 1 \\
$s_{3}$ & 0 & 0 & 1 & 1 & 0 & 0 & 0 \\
$s_{4}$ & 0 & 1 & 0 & 0 & 1 & 0 & 0
\end{tabular}

Through investigation and calculation of these four enterprises $s_{1}, s_{2}, s_{3}, s_{4}$, the investment bank gets the exact number as shown in Table 1. $a_{1}, a_{2}, a_{3}$ are the benefit type indexes and $a_{4}$ is the cost type indexes.

Calculated using (1): $[1.6,2.0] \preceq[1.8,2.2] \preceq[2.0,2.4] \preceq[2.3,2.7]$, we have $f_{\otimes}\left(S, a_{1}\right)=\left\{a_{11}, a_{12}, a_{13}, a_{14}\right\}=\{[1.6,2.0],[1.8,2.2],[2.0,2.4],[2.3,2.7]\}$, where $S=\left\{s_{1}, s_{2}, s_{3}, s_{4}\right\}$. Similarly, the followings hold: $f_{\otimes}\left(S, a_{1}\right)=\left\{a_{21}, a_{22}, a_{23}, a_{24}\right\}=$ $\{[1.2,1.8],[1.5,2.1],[1.7,2.3],[2.4,3.0]\} ; f_{\otimes}\left(S, a_{3}\right)=\left\{a_{31}, a_{32}, a_{33}\right\}=\{[1.6,2.0]$, $[1.8,2.2],[1.9,2.3]\} ; f_{\otimes}\left(S, a_{4}\right)=\left\{a_{41}, a_{42}, a_{43}, a_{44}\right\}=\{[4.4,4.6],[4.9,5.1],[5.4,5.6]$, $[6.4,6.6]\}$.

Step 1. Determine the grey formal context with original decision table Table 1 and find all formal concepts in it.

The standardized grey formal context is shown in Tables 2 and 3.

Obtain the formal concepts from Tables 2 and 3 :

$\left(\{1\},\left\{a_{11}, a_{12}, a_{21}, a_{31}, a_{32}, a_{41}, a_{42}, a_{43}\right\}\right)$,

$\left(\{2\},\left\{a_{11}, a_{12}, a_{13}, a_{14}, a_{21}, a_{22}, a_{23}, a_{24}, a_{31}, a_{41}, a_{42}, a_{43}, a_{44}\right\}\right)$, 
$\left(\{3\},\left\{a_{11} a_{21}, a_{22}, a_{23}, a_{31}, a_{32}, a_{33}, a_{41}\right\}\right)$,

$\left(\{4\},\left\{a_{11}, a_{12}, a_{13}, a_{21}, a_{22}, a_{31}, a_{32}, a_{41}, a_{42}\right\}\right)$,

$\left(\{1,2\},\left\{a_{11}, a_{12}, a_{21}, a_{31}, a_{41}, a_{42}, a_{43}\right\}\right)$,

$\left(\{1,3\},\left\{a_{11}, a_{21}, a_{31}, a_{32}, a_{41}\right\}\right)$,

$\left(\{1,4\},\left\{a_{11}, a_{12}, a_{21}, a_{31}, a_{32}, a_{4} 1, a_{42}\right\}\right)$,

$\left(\{2,3\},\left\{a_{11}, a_{21}, a_{22}, a_{23}, a_{31}, a_{41}\right\}\right)$,

$\left(\{2,4\},\left\{a_{11}, a_{12}, a_{13}, a_{21}, a_{22}, a_{31}, a_{41}, a_{42}\right\}\right)$,

$\left(\{3,4\},\left\{a_{11}, a_{21}, a_{22}, a_{31}, a_{32}, a_{41}\right\}\right)$,

$\left(\{1,2,3\},\left\{a_{11}, a_{21}, a_{31}, a_{41}\right\}\right)$,

$\left(\{1,2,4\},\left\{a_{11}, a_{12}, a_{21}, a_{31}, a_{41}, a_{42}\right\}\right)$,

$\left(\{1,3,4\},\left\{a_{11}, a_{21}, a_{31}, a_{32}, a_{41}\right\}\right)$,

$\left(\{2,3,4\},\left\{a_{11}, a_{21}, a_{22}, a_{31}, a_{41}\right\}\right)$,

$\left(\{1,2,3,4\},\left\{a_{11}, a_{21}, a_{31}, a_{41}\right\}\right)$.

We select the following grey concepts to determine the best option.

$\left(\{1\},\left\{a_{11}, a_{12}, a_{21}, a_{31}, a_{32}, a_{41}, a_{42}, a_{43}\right\}\right)$,

$\left(\{2\},\left\{a_{11}, a_{12}, a_{13}, a_{14}, a_{21}, a_{22}, a_{23}, a_{24}, a_{31}, a_{41}, a_{42}, a_{43}, a_{44}\right\}\right)$,

$\left(\{3\},\left\{a_{11} a_{21}, a_{22}, a_{23}, a_{31}, a_{32}, a_{33}, a_{41}\right\}\right)$,

$\left(\{4\},\left\{a_{11}, a_{12}, a_{13}, a_{21}, a_{22}, a_{31}, a_{32}, a_{41}, a_{42}\right\}\right)$.

Step 2. Normalization. Use (10) to (13) to normalize the corresponding objective series , thus each feature has the same degree of influence and the method cannot be affected by the choice of units and scales. Table 4 contains the normalized results.

Step 3. Data series construction. View each of the row vectors of the matrix as a data series, and obtain a total of $n$ series. These series are:

$\otimes \mathbf{u}^{+}=([0.6363,1],[0.6667,1],[0.4285,1],[0.909,1])$,

$\otimes \mathbf{u}^{-}=([0,0.3636],[0,0.3333],[0,0.5714],[0,0.0909])$.

Step 4. Grey relational coefficient. In this case, adopt $\lambda=1$, compute $\Delta$ before getting the coefficient. The process is showed at Table 5. We obtain that $\Delta_{\min }^{+}=0$, $\underline{\Delta}_{\max }^{+}=0.909$. Using similar method, there are $\bar{\Delta}_{\min }^{+}=0, \bar{\Delta}_{\max }^{+}=0.909, \underline{\Delta}_{\text {min }}^{-}=0$, $\underline{\Delta}_{\max }^{-}=0.909, \bar{\Delta}_{\min }^{-}=0, \bar{\Delta}_{\max }^{-}=0.909$.

Calculate the grey relational coefficient $r_{i j}^{+}$and $r_{i j}^{-}$between $\otimes u_{i j}$ and the two different factors by (17) and (19) (see Tables 6 and 7).

Step 5. Grey relational grade calculation.

Table 4

Normalization decision matrix that is constructed by object set $\left\{s_{1}\right\},\left\{s_{2}\right\},\left\{s_{3}\right\},\left\{s_{4}\right\}$ of grey formal concepts

\begin{tabular}{lllll}
\hline & $a_{1}$ & $a_{2}$ & $a_{3}$ & $a_{4}$ \\
\hline$\left\{s_{1}\right\}$ & {$[0.1818,0.5454]$} & {$[0,0.3333]$} & {$[0.2857,0.8571]$} & {$[0.4545,0.5454]$} \\
$\left\{s_{2}\right\}$ & {$[0.6363,1]$} & {$[0.6667,1]$} & {$[0,0.5714]$} & {$[0,0.0909]$} \\
$\left\{s_{3}\right\}$ & {$[0,0.3636]$} & {$[0.2778,0.6111]$} & {$[0.4285,1]$} & {$[0.9090,1]$} \\
$\left\{s_{4}\right\}$ & {$[0.3636,0.7272]$} & {$[0.1667,0.5]$} & {$[0.2867,0.8571]$} & {$[0.6818,0.7727]$} \\
\hline
\end{tabular}


Table 5

Computing $\underline{\Delta}_{\min }^{+}$and $\underline{\Delta}_{\max }^{+}$

\begin{tabular}{lllllll}
\hline & $a_{1}$ & $a_{2}$ & $a_{3}$ & $a_{4}$ & $\min _{j}\left\{\underline{\Delta}^{+}\right\}$ & $\max _{j}\left\{\underline{\Delta}^{+}\right\}$ \\
\hline$\underline{\Delta}^{+}(1)$ & 0.4545 & 0.6667 & 0.1248 & 0.4545 & 0.1248 & 0.6667 \\
$\underline{\Delta}^{+}(2)$ & 0 & 0 & 0.4285 & 0.909 & 0 & 0.909 \\
$\underline{\Delta}^{+}(3)$ & 0.6363 & 0.3889 & 0 & 0 & 0 & 0.6363 \\
$\underline{\Delta}^{+}(4)$ & 0.2727 & 0.5 & 0.1248 & 0.2272 & 0.1428 & 0.5 \\
$\underline{\Delta}_{\min }^{+}$ & & & & & 0 & \\
$\underline{\Delta}_{\max }^{+}$ & & & & & & 0.909 \\
\hline
\end{tabular}

Table 6

Grey relational coefficient matrix $r_{i j}^{+}$

$$
r_{i j}^{+}=\left[\begin{array}{llll}
0.5 & 0.4053 & 0.7609 & 0.5 \\
1 & 1 & 0.5147 & 0.3333 \\
0.4167 & 0.5388 & 1 & 1 \\
0.625 & 0.4761 & 0.7609 & 0.6667
\end{array}\right] .
$$

Table 7

Grey relational coefficient matrix $r_{i j}^{-}$

$$
r_{i j}^{+}=\left[\begin{array}{llll}
0.7142 & 1 & 0.6140 & 0.5 \\
0.4167 & 0.4053 & 1 & 1 \\
1 & 0.6206 & 0.5147 & 0.3333 \\
0.5556 & 0.7316 & 0.6140 & 0.3999
\end{array}\right]
$$

$$
\begin{array}{ll}
G\left(\otimes \mathbf{u}^{+}, \otimes u_{1}\right)=0.5042, & G\left(\otimes \mathbf{u}^{+}, \otimes u_{2}\right)=0.7388, \\
G\left(\otimes \mathbf{u}^{+}, \otimes u_{3}\right)=0.6322, & G\left(\otimes \mathbf{u}^{+}, \otimes u_{4}\right)=0.7120 ; \\
G\left(\otimes \mathbf{u}^{-}, \otimes u_{1}\right)=0.7071, & G\left(\otimes \mathbf{u}^{-}, \otimes u_{2}\right)=0.6172, \\
G\left(\otimes \mathbf{u}^{-}, \otimes u_{3}\right)=0.5752, & G\left(\otimes \mathbf{u}^{-}, \otimes u_{4}\right)=0.7055 .
\end{array}
$$

Step 6. Feature subset selection. $G\left(\otimes \mathbf{u}_{1}\right)=0.3986, G\left(\otimes \mathbf{u}_{2}\right)=0.5609, G\left(\otimes \mathbf{u}_{3}\right)=$ $0.5285, G\left(\otimes \mathbf{u}_{4}\right)=0.5033$. So get the same order of business with the literature (Wang et al., 2009b): $\left\{s_{2}\right\} \succ\left\{s_{3}\right\} \succ\left\{s_{4}\right\} \succ\left\{s_{1}\right\}$. 


\section{Conclusions and Discussions}

In this paper, we have addressed the issues of lattice structure of data sets in a grey information system. A dominance relation definition of grey number has been given. Under this definition, it has been proved that different grey number of the same attributes is comparable. Based on the grey dominance relation defined in this paper, two posets have been discussed. Then, the definition and building method of concept lattice based on grey dominance relations were proposed. Theoretical studies and numerical experiments have been carried out to show that the method are effective and suitable to deal with uncertainty and discovery rules in a decision table, and have a much simpler and more comprehensive form than the existing ones. These may be helpful for knowledge discovery.

Acknowledgements. The authors wish to thank the anonymous reviewers for their constructive comments.

\section{References}

Chang, S.H., Wu, J.H., Li, Z. (1996). Frequency synthesizer product or/and process optimization using the grey rational analysis. The Journal of Grey System, 8(3), 235-260.

Deng, J.L. (1982). Control problems of grey systems. Systems and Control Letters, 1(5), 288-294.

Deng, J.L. (1989). Introduction to grey system theory. The Journal of Grey Theory, 1, 1-24.

Deng, J.L. (1990). A Course on Grey System Theory. Huazhong University of Science and Technology Press, Wuhan.

Ganter, B., Wille, R. (1999). Formal Concept Analysis: Mathematical Foundations. Berlin, Springer.

Huang, S.J., Chiu, N.H., Chen, L.W. (2008). Integration of the grey relational analysis with genetic algorithm for software effort estimation. European Journal of Operational Research, 188(3), 898-909.

Liang, J.Y., Wang, J.H. (2004). An algorithm for extracting rules generating set based on concept lattice. Journal of Computer Research and Development. 41(8), 1339-1344.

Li, G.D., Yamaguchi, D., Nagai, M. (2007). Application of grey-based rough decision-making approach to suppliers selection. Journal of Modelling in Management, 2(02), 131-137.

Li, G.D., Yamaguchi, D., Nagai, M. (2008). A grey-based rough decision-making approach to supplier selection. The International Journal of Advanced Manufacturing Technology, 36(9/10), 1032-1040.

Lin, Y.H., Lee, P.C. (2007). Novel high-precision grey forecasting model. Automation in Construction, 16(6), 771-777.

Lin, Y., Chen, M.Y., Liu, S. (2004). Theory of grey systems: capturing uncertainties of grey information. Kybernetes, 33(2), 196-218.

Lin, Y.H., Lee, P.C., Chang, T.P., Ting, H.I. (2008). Multi-attribute group decision making model under the condition of uncertain information. Automation in Construction, 17(6), 792-797.

Liu, S., Lin, Y. (2006). On measures of information content of grey numbers. Kybernetes, 35(6), 899-904.

Mi, C.M., Liu, S.F., Fang, Z.G., Wu, X., Zhang, H.W. (2006). The driver's shortest path decision-making model and its algorithm study based on grey number superiority relationship. In: Proceedings of the Fifth International Conference on Machine Learning and Cybernetics, Dalian, pp. 709-714.

Noorul, H.A., Kannan, G. (2007). A hybrid normalised multi criteria decision making for the vendor selection in a supply chain model. International Journal Management and Decision Making, 8(5/6), 601-622.

Olson, D.L., Wu, D. (2006). Simulation of fuzzy multiattribute models for grey relationships. European Journal of Operational Research, 175(1), 111-120.

Qu, K.S., Zhai, Y.H. (2006). Posets, inclusion degree theory and FCA. Chinese journal of computers, 29, 219226.

Shi, J.R., Liu, S.Y., Xiong, W.T. (2005). A new solution for interval number linear programming. Journal of Systems Engineering Theoryand Practice, 2, 101-106 (in Chinese). 
Tonella, P. (2003). Using a concept lattice of decomposition slices for program understanding and impact analysis. IEEE Transactions on Software Engineering, 29(6), 495-509.

Trotter, W.T. (1995). Partially ordered sets. In: Graham, R.L., GrOtschel, M., Lovfisz, L. (Eds.), Handbook of Combinatorics, Vol. 1. Elsevier, Amsterdam, pp. 433-480.

Valtchev, P., Missaoui, R., Godin, R., Meridji, M. (2002). Generating frequent itemsets incrementally two novel approaches based on galois lattice theory. Journal of Experimental and Theoretical Artificial Intelligence, $14(2 / 3), 115-142$.

Wang, J.H., Liang, J.Y., Qu, K.S. (2009a). Concept lattice based on dominance relations. Computer Science, 36(7), 161-163, 201.

Wang, Z.X., Dang, Y.G., Wei, J., Yang, H. (2009b). Study on the extending multi-attribute decision model of grey target. Journal of Systems Engineering and Electronics, 20(5), 985-991.

Wille, R. (1982). Restructuring lattice theory: an approach based on hierarchies of concepts. In: Rival, I. (Ed.), Ordered Sets. Reidel, Dordreeht, pp. 445-470.

Wu, Q., Liu, Z.T. (2009). Real formal concept analysis based on grey-rough set theory. Knowledge-Based Systems, 22(1), 38-45.

Wu, H.S., Deng, J.L., Win, K.L. (1996). Introduction to Grey Theory, Gao-Li Co, Taipei, Taiwan (in Chinese).

Xie, N.M., Liu, S.F. (2011). A novel grey relational model based on grey number sequences. Grey Systems: Theory and Application, 1(2), 117-128.

Zavadskas, E.K., Kaklauskas, A., Turskis, Z., Tamosaitiene, J. (2009). Multi-attribute decision-making model by applying grey numbers. Informatica, 20(2), 305-320.

Q. Wu graduated from the Dalian University of Technology in 1999 and received a master degree in informatics. In 2008, He received a doctoral degree in computer science $(\mathrm{PhD})$ from Shanghai University. He is an associate professor at the Shaoxing University. Her research interests include data mining, formal concept analysis, and internet technologies.

\section{Pilkosios gardelių sampratos sudarymas ir taikymas}

Qiang WU

Pilkieji skaičiai padeda išreikšti ne tik atskiru aibès elementŭ, bet ir aibės kaip visumos neapibrežtumą. Šiame straipsnyje neapibrežtai informacijai ir kiekvienos originalios sekos neapibrèžtumo laipsniui perteikti sujungiama tikimybès laipsnio samprata iš pilkuju sistemu teorijos ir dominavimo santykiai bei iš dalies sutvarkytos aibės iš šiurkščiuju aibių teorijos. Parengta pilkuju informacinių sistemu gardeliu samprata ir aprašytas sprendimu prièmimo algoritmas, taikant pilkuosius santykius.Pateikta atvejo analizè, kuomet pasiūlytas metodas taikomas tiekèjams parinkti. Atlikti tyrimai parodè, jog šis metodas yra tinkamas naudoti. 